\title{
Anti-PD-I Immunotherapy Improves the Efficacy of Hepatic Artery Infusion Chemotherapy in Advanced Hepatocellular Carcinoma
}

\author{
This article was published in the following Dove Press journal: \\ Journal of Hepatocellular Carcinoma
}

\author{
Jie $M \mathrm{Mi}^{1,2, *}$ \\ Shao-Hua $\mathrm{Li}^{1,2, *}$ \\ Qi-jiong $\mathrm{Li}^{1,2, *}$ \\ Xu-Qi Sun ${ }^{1,2}$ \\ Liang-He Lu ${ }^{1,2}$ \\ Wen-Ping Lin ${ }^{1,2}$ \\ Lie Zheng (D) ${ }^{1,3}$ \\ Min-Shan Chen ${ }^{1,2}$ \\ Ming Shi ${ }^{1,2}$ \\ Wei Wei ${ }^{1,2}$ \\ Rong-Ping Guo (D) 1,2 \\ 'Sun Yat-Sen University Cancer Center, \\ State Key Laboratory of Oncology in \\ South China, Collaborative Innovation \\ Center for Cancer Medicine, Guangzhou, \\ 510060, People's Republic of China; \\ ${ }^{2}$ Department of Liver Surgery, Sun Yat- \\ Sen University Cancer Center, \\ Guangzhou, 510060, People's Republic of \\ China; ${ }^{3}$ Department of Medical Imaging, \\ Sun Yat-Sen University Cancer Center, \\ Guangzhou, 510060, People's Republic of \\ China
}

*These authors contributed equally to this work
Background: Hepatic artery infusion chemotherapy (HAIC) and anti-programmed cell death protein-1 (PD-1) immunotherapy have shown promising outcomes in patients with advanced hepatocellular carcinoma (HCC), respectively. However, the combination of the two treatments has not been reported. In this study, we compared the efficacy of HAIC combined with anti-PD-1 immunotherapy (HAICAP) and HAIC in patients with advanced HCC.

Methods: Between November 2018 and December 2019, advanced HCC patients that were treated with either HAICAP or HAIC were retrospectively recruited and reviewed for eligibility. Efficacy was evaluated according to tumor response and survival.

Results: As a result, 229 patients were included in this study. Patients were divided into HAICAP group $(n=81)$ and HAIC group $(n=148)$ accordingly. The follow-up time ranged from 1.0 to 21.6 months, with a median of 11.0 months. The median overall survival was 18.0 months in the HAICAP group and 14.6 months in the HAIC group $(\mathrm{p}=0.018$; HR $=$ $0.62 ; 95 \%$ CI $0.34-0.91)$. The median progression-free survival was 10.0 months in the HAICAP group and 5.6 months in the HAIC group $(\mathrm{p}=0.006 ; \mathrm{HR}=0.65 ; 95 \% \mathrm{CI}$ $0.43-0.87)$. The disease control rate in overall response ( $83 \%$ vs $66 \%$; $=0.006)$ and intrahepatic response ( $85 \%$ vs $74 \%$, respectively; $p=0.045)$ were higher in the HAICAP group than in the HAIC group.

Conclusion: In comparison to HAIC, HAICAP was associated with a better treatment response and survival benefits for patients with advanced HCC.

Keywords: hepatocellular carcinoma, hepatic artery infusion chemotherapy, programmed cell death protein-1, FOLFOX, combination therapy

\section{Introduction}

Hepatocellular carcinoma (HCC) is one of the most common malignancies and the fourth leading cause of cancer-related deaths worldwide. ${ }^{1}$ Unfortunately, due to its uneventful onset, HCC often progresses to an advanced stage before it can be detected, at which point surgical resection is not possible. In these cases, Barcelona guidelines recommend oral sorafenib as the first-line treatment. ${ }^{2}$ However, the outcome of these patients is barely improved, with a median survival time of 6.5 to 10.7 months. ${ }^{2,3}$ Hepatic artery infusion chemotherapy (HAIC) is an emerging therapy that has attracted much attention due to its high response rates and favorable survival for advanced HCC. ${ }^{4,5}$ HAIC has been widely applied as an alternative therapy to sorafenib for advanced HCC patients in the Asian region. ${ }^{6-8}$ As
Correspondence: Rong-Ping Guo;

Wei Wei

Email guorp@sysucc.org.cn;

weiwei@sysucc.org.cn 
a locoregional interventional therapy, HAIC showed better efficacy and safety in the treatment of unresectable HCC than conventional transarterial chemoembolization. ${ }^{6,9-11}$ Although HAIC had many benefits, it showed unsatisfying control ability on extrahepatic metastases, indicating the efficacy remains limited and needs to be further improved. $^{12}$

Programmed cell death protein-1 (PD-1) inhibitors are currently the focus of cancer research. The survival benefit of PD-1 inhibitors in unresectable HCC patients is beyond borders, with a median OS of 13.9-15.6 months, and its treatment-related toxic effects are relatively low. ${ }^{13,14}$ Recently, several studies reported the efficacy of PD-1 inhibitors in combination with tyrosine kinase inhibitors or locoregional therapies in the comprehensive treatment of advanced HCC. ${ }^{15,16}$ As a systemic therapy, anti-PD-1 immunotherapy is expected to have great potential in combination therapy.

To the best of our knowledge, the efficacy of HAIC in combination with anti-PD-1 immunotherapy (HAICAP) in advanced HCCs has never been reported. In this study, we investigate the potential of anti-PD-1 immunotherapy as a combination therapy of HAIC. We designed this retrospective study to compare the outcome of advanced HCC patients with HAICAP or HAIC monotherapy, aiming to provide a reference for the treatment of advanced HCC.

\section{Materials and Methods}

This study was conducted according to the ethical guidelines of the 1975 Declaration of Helsinki. The analysis of patient data was reviewed and approved by the Institutional Review Board and Human Ethics Committee at the Sun Yat-sen University Cancer Center (RDDA2020001831, SYSUCC, Guangzhou, China).

\section{Patients}

Between November 2018 and December 2019, the medical records of patients diagnosed with $\mathrm{HCC}$ who received HAICAP and HAIC treatment at the Department of Liver Surgery of SYSUCC were reviewed for eligibility. Patients were included based on the following specific criteria: (a) patients were diagnosed with HCC through imaging or pathology according to the AASLD practice guidelines; ${ }^{17}$ (b) patients had at least one cycle of HAICAP or HAIC; (c) no cancer-related therapies were involved before or during HAICAP or HAIC; (d) patients had a tumor classification of Barcelona Clínic Liver Cancer (BCLC) B or C; (e) Child-Pugh (CP) was classified as A; (f) patients aged 18 to 75 ; (g) no other malignant tumors were diagnosed; and (h) complete medical and follow-up data were available. All laboratory serum test data were collected within 3 days before the initial treatment. Imaging evaluation included enhanced computed tomography (CT) or magnetic resonance imaging (MRI) examination within a week before the initial treatment.

\section{Treatment Procedure}

HAIC was administered according to previously described procedures. ${ }^{18}$ Femoral artery puncture and catheterization were performed in every cycle of treatment. The FOLFOX regimen was administered via the hepatic artery as follows: 85 or $135 \mathrm{mg} / \mathrm{m}^{2}$ oxaliplatin, $400 \mathrm{mg} / \mathrm{m}^{2}$ leucovorin, and $400 \mathrm{mg} / \mathrm{m}^{2}$ fluorouracil on the first day; and $2400 \mathrm{mg} /$ $\mathrm{m}^{2}$ fluorouracil over 46 hours. For HAICAP group, patients received anti-PD-1 agents within 2 days before or after the start of HAIC. Anti-PD-1 agents were used intravenously at the standard dose (Supplementary Table

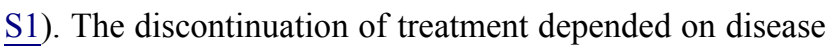
progression, unacceptable toxicity, patient withdrawal of consent, or changes of treatment plan. The final follow-up ended on November 30, 2020. Enhanced CT or MRI was performed every 4-6 weeks after treatment begins. Routine follow-up intervals were 2-4 months.

\section{Diagnosis and Definitions}

Tumor response was defined as complete response (CR), partial response (PR), stable disease (SD), or progressive disease (PD) according to the modified Response Evaluation Criteria in Solid Tumors 1.1 (mRECIST). ${ }^{19}$ Treatment-related adverse events (AEs) were evaluated by National Cancer Institute Common Terminology Criteria for Adverse Events version 4.0. Overall response rate (ORR) was calculated as the sum of $C R$ and $P R$. Disease control rate (DCR) was calculated as the sum of $\mathrm{CR}, \mathrm{PR}$, and SD. Overall survival (OS) was defined as the time interval from treatment initiation to cancer-related death. Progression-free survival (PFS) was defined as the time interval from treatment initiation to progression or death.

\section{Statistical Analysis}

Categorical variables in the baseline characteristics were compared using the Pearson's $\chi^{2}$ test or Fisher's exact test. Survival analysis was calculated using the Kaplan-Meier method, and differences in the survival curves were 


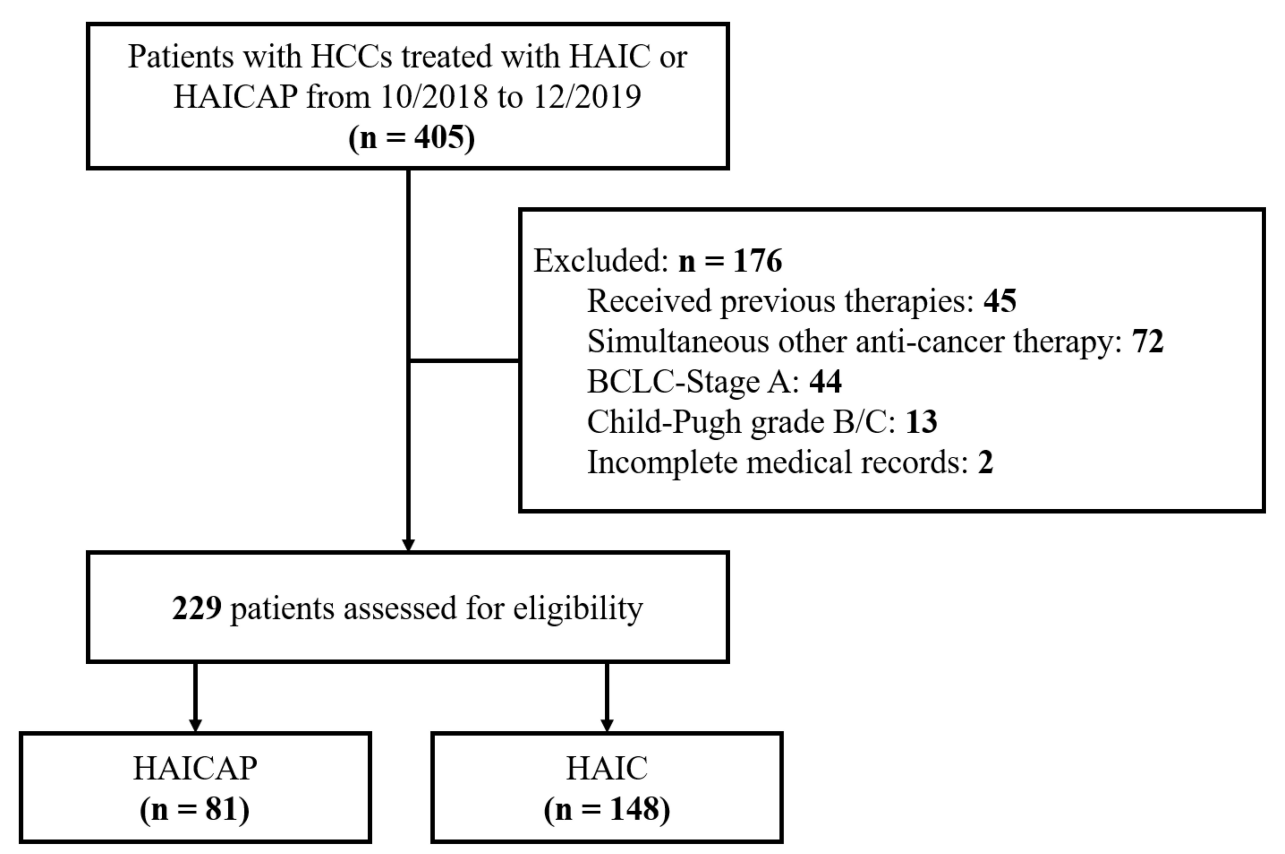

Figure I Flow diagram summarizing the disposition process of patients.

analyzed with a Log rank test. All variables with a $\mathrm{P}$ value $<0.05$ in univariate analyses were used in multivariate analyses using Cox regression models. The hazard ratio (HR) and confidence intervals (CI) were calculated. A two-tailed $\mathrm{P}$ value $<0.05$ was considered statistically significant. All data analyses were performed using SPSS 25.0 software (SPSS Inc., Chicago, IL) and $\mathrm{R}$ version 4.0.2.

\section{Result}

\section{Patient Characteristics and Treatments}

From November 2018 to December 2019, 405 patients with HCCs who received HAICAP or HAIC were assessed. The following cases were excluded: 45 patients received previous surgery, interventional therapies, tyrosine kinase inhibitors or immune-targeted therapies; 72 patients participated in other treatments during HAICAP or HAIC; 44 patients were classified with a tumor grade of BCLC/A; 13 patients were classified as $\mathrm{CP} \mathrm{B}$ or $\mathrm{C}$; and 2 patients had missing sections in their medical records. Finally, a total of 229 patients were included in the study, and the patients were divided into HAICAP group $(n=81)$ or HAIC group $(n=148)$. The patient characterization process is shown in Figure 1. Of note, HAICAP was conducted in July 2018 in our center.
The clinical characteristics and treatment of patients are summarized in Table 1. A total of 229 Asian patients were included, $206(90 \%)$ of whom were male. Most patients were with $\mathrm{CP}$ score of $5(82 \%$ in the two groups) and BCLC/C (68\% in the HAICAP group and $62 \%$ in the HAIC group). Two groups were comparable in clinical characteristics, liver function and tumor burden. PD-1 inhibitor categories are summarized in Table $\underline{\mathrm{S} 1}$. The cycles of HAIC in the HAIC group ranged from 1 to 8 (with a median of 3 ) and ranged from 1 to 6 in the HAICAP group (with a median of 3). The cycles of anti-PD-1 therapy in the HAICAP group ranged from 1 to 14 (with a median of 5). More patients in the HAICAP group received subsequent surgical resection than the HAIC group $(17.3 \%$ vs $8.1 \%, \mathrm{P}=0.036)$. Details of the follow-up treatment are listed in Supplementary Table S2.

\section{Survival}

The follow-up time ranged from 1.0 to 21.6 months, with a median of 11.0 months. Patients in the HAICAP group had significantly better survival outcomes than those in the HAIC group. The 6-, 12- and 18-month OS was 97.5\%, 80.4\% and 55.1\%, respectively, in the HAICAP group, and $91.7 \%, 61.9 \%$ and 
Table I Baseline Clinical Characteristics of Patients

\begin{tabular}{|c|c|c|c|}
\hline Characteristics $^{\mathbf{a}}$ & HAICAP $(n=8 I)$ & HAIC $(n=\mid 48)$ & $P$ value \\
\hline Age (years) & & & 0.111 \\
\hline$\leq 50$ & $30(37)$ & $71(48)$ & \\
\hline$>50$ & $5 I(63)$ & $77(52)$ & \\
\hline Gender & & & 0.602 \\
\hline Female & $7(9)$ & $16(11)$ & \\
\hline Male & $74(9 I)$ & I 32 (89) & \\
\hline $\mathrm{HBsAg}$ & & & 0.691 \\
\hline Negative & $9(11)$ & $14(9)$ & \\
\hline Positive & $72(89)$ & $134(9 \mid)$ & \\
\hline HBV-DNA & & & 0.883 \\
\hline$\leq 10^{3}$ & $32(40)$ & $57(39)$ & \\
\hline$>10^{3}$ & $49(60)$ & $91(6 I)$ & \\
\hline Liver cirrhosis & & & 0.526 \\
\hline No & $33(4 I)$ & $54(36)$ & \\
\hline Yes & $48(59)$ & $94(64)$ & \\
\hline NLR & & & 0.915 \\
\hline$\leq 3$ & $4 \mid(5 I)$ & $76(5 I)$ & \\
\hline$>3$ & $40(49)$ & $72(49)$ & \\
\hline PLT (I0E9/L) & & & 0.176 \\
\hline$\leq 100$ & $9(11)$ & $9(6)$ & \\
\hline$>100$ & $72(89)$ & $139(94)$ & \\
\hline ALT (U/L) & & & 0.159 \\
\hline$\leq 50$ & $37(46)$ & $82(55)$ & \\
\hline$>50$ & $44(54)$ & $66(45)$ & \\
\hline AST (U/L) & & & 0.197 \\
\hline$\leq 40$ & $29(36)$ & $66(45)$ & \\
\hline$>40$ & $52(64)$ & $82(55)$ & \\
\hline $\operatorname{AFP}(\mathrm{ng} / \mathrm{mL})$ & & & 0.709 \\
\hline$\leq 400$ & $36(44)$ & $62(42)$ & \\
\hline$>400$ & $45(56)$ & $86(58)$ & \\
\hline $\mathrm{DCP}(\mathrm{ng} / \mathrm{mL})$ & & & 0.977 \\
\hline$\leq 400$ & $16(20)$ & $29(20)$ & \\
\hline$>400$ & $65(80)$ & $119(80)$ & \\
\hline Child-Pugh score & & & 0.858 \\
\hline 5 & $66(82)$ & $122(82)$ & \\
\hline 6 & $15(18)$ & $26(18)$ & \\
\hline $\mathrm{BCLC}$ stage & & & 0.386 \\
\hline B & $26(32)$ & $56(38)$ & \\
\hline C & $55(68)$ & $92(62)$ & \\
\hline Tumor number & & & 0.977 \\
\hline Single & $16(20)$ & $29(20)$ & \\
\hline Multiple & $65(80)$ & $119(80)$ & \\
\hline
\end{tabular}

(Continued) 
Table I (Continued).

\begin{tabular}{|c|c|c|c|}
\hline Characteristics $^{\mathrm{a}}$ & HAICAP $(n=8 I)$ & HAIC $(n=148)$ & $P$ value \\
\hline Tumor distribution & & & 0.124 \\
\hline Uni-lobar & $48(59)$ & $72(49)$ & \\
\hline Bi-lobar & $33(4 I)$ & $76(5 I)$ & \\
\hline Size of largest nodule $(\mathrm{cm})$ & & & 0.544 \\
\hline$\leq 5$ & $9(11)$ & $15(10)$ & \\
\hline$<5-10$ & $30(37)$ & $55(37)$ & \\
\hline$<10-15$ & $29(36)$ & $63(43)$ & \\
\hline$>15$ & $13(16)$ & $15(10)$ & \\
\hline Tumor thrombus ${ }^{\mathrm{b}}$ & & & 0.112 \\
\hline None & $29(36)$ & $68(45)$ & \\
\hline$V_{p l}-2$ & $22(27)$ & $22(15)$ & \\
\hline$V_{p 3}$ & $22(27)$ & $38(26)$ & \\
\hline$V_{p} 4$ & $8(10)$ & $20(14)$ & \\
\hline Extrahepatic metastasis & & & 0.147 \\
\hline Absence & $67(83)$ & $110(74)$ & \\
\hline Presence & $14(17)$ & $38(26)$ & \\
\hline
\end{tabular}

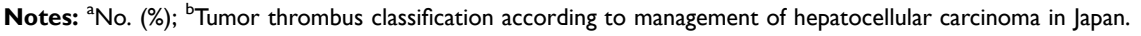

Abbreviations: HAIC, hepatic infusion chemotherapy; HAICAP, hepatic infusion chemotherapy plus anti-programmed cell-death-protein-I agent; HBsAg, hepatitis B surface antigen; HBV, hepatitis B virus; NLR, neutrophil-lymphocyte ratio; PLT, blood platelet; ALT, alanine aminotransferase; AST, aspartate aminotransferase; AFP, alphafetoprotein; DCP, des-gamma-carboxy prothrombin; BCLC, Barcelona Clinic Liver Cancer.

$41.3 \%$, respectively, in the HAIC group. The median OS was 18.0 months in the HAICAP group and 14.6 months in the HAIC group $(\mathrm{p}=0.018 ; \mathrm{HR}=0.62 ; 95 \% \mathrm{CI}$ $0.34-0.91)$. The 3-, 6- and 12- PFS was 84.6\%, 66.8\% and $41.8 \%$, respectively, in the HAICAP group, and $70.2 \%, 47.5 \%$ and $26.3 \%$, respectively, in the HAIC group. The median PFS was 10.0 months in the HAICAP group and 5.6 months in the HAIC group (p
$=0.006 ; \mathrm{HR}=0.65 ; 95 \%$ CI $0.43-0.87)$. The survival curves are shown in Figure 2. The forest plot analysis of factors associated with OS and PFS is shown in Figure 3. Generally, compared to HAIC, HAICAP provided a clinical benefit in patients with large, multiple, bilateral HCCs, but failed to have a survival benefit in patients with main portal vein tumor thrombus or extrahepatic metastasis.
A

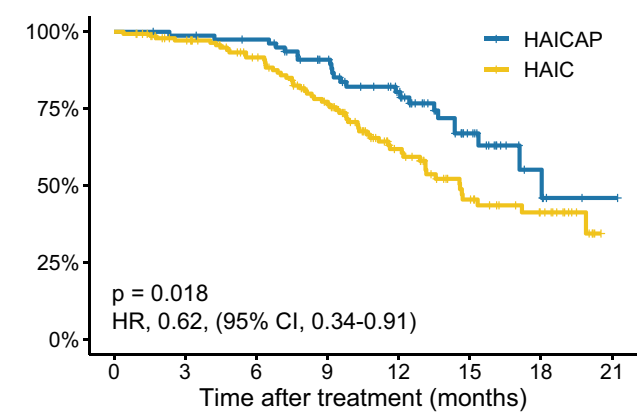

Number at risk

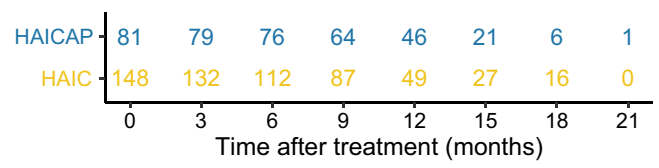

B

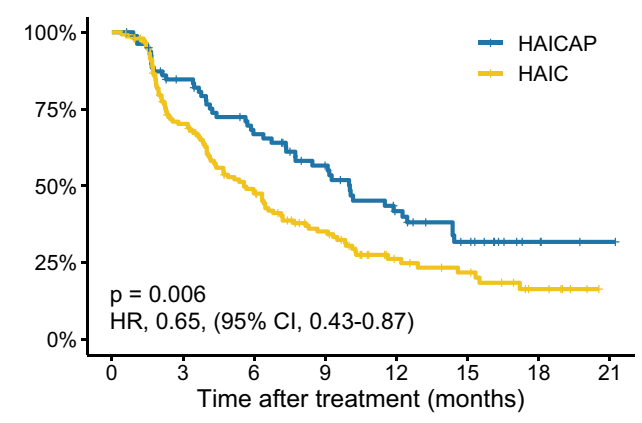

Number at risk

$$
\text { HAICAP }\left\{\begin{array}{cccccccc}
81 & 63 & 48 & 36 & 23 & 14 & 4 & 1 \\
148 & 96 & 63 & 39 & 19 & 14 & 6 & 0 \\
\hline 0 & 3 & 6 & 9 & 12 & 15 & 18 & 21
\end{array}\right.
$$

Figure 2 Kaplan-Meier curves of survival outcomes of patients in the two groups. (A) Overall survival. (B) Progression-free survival.

Abbreviations: HAIC, hepatic artery infusion chemotherapy; HAICAP, hepatic artery infusion chemotherapy combined with anti-PD-I immunotherapy. 


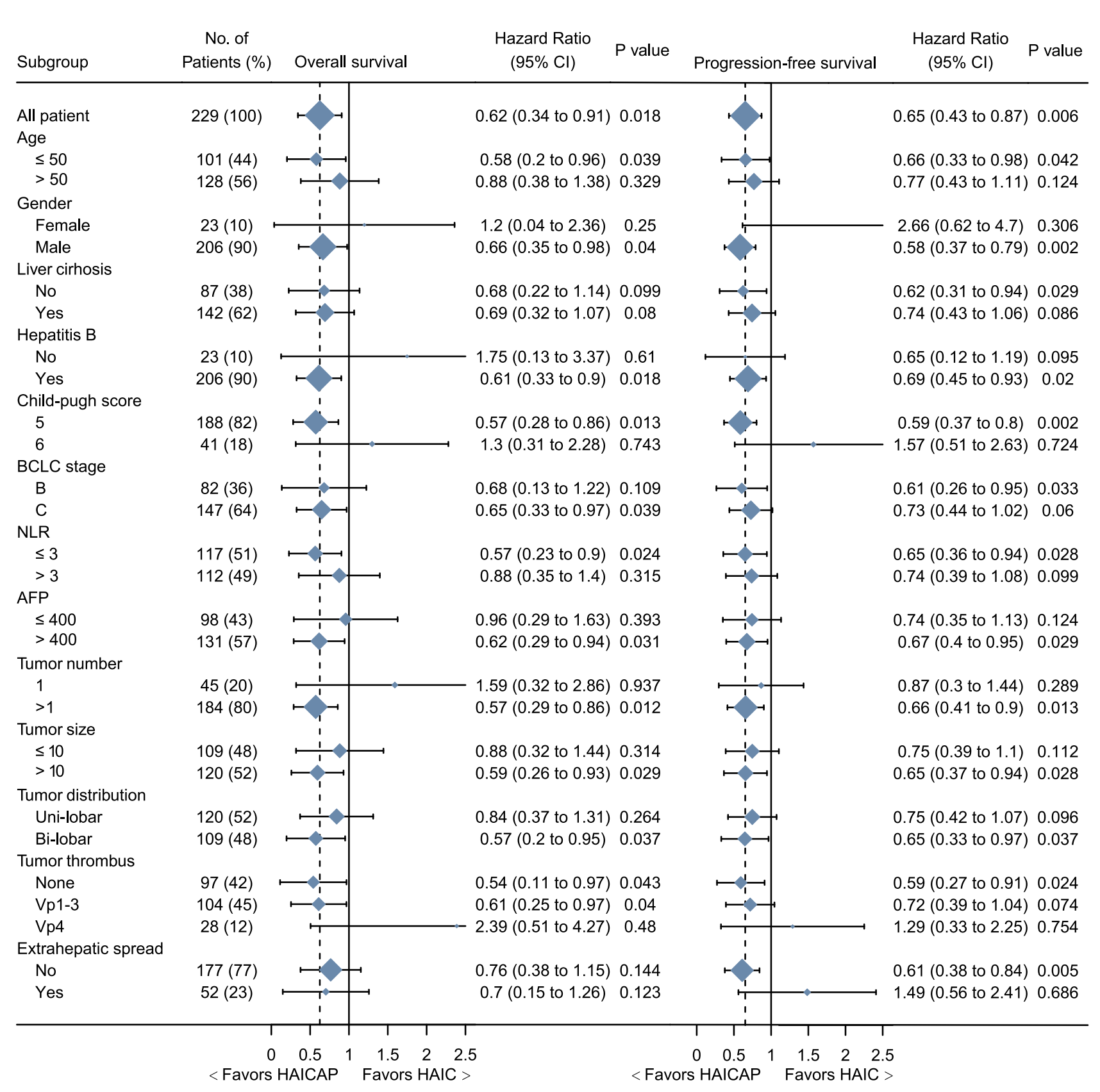

Figure 3 Forest plot for overall survival and progression-free survival of the whole cohort.

Abbreviations: HAIC, hepatic artery infusion chemotherapy; HAICAP, hepatic artery infusion chemotherapy combined with anti-PD-I immunotherapy.

\section{Tumor Response}

The treatment response is summarized in Table 2. Based on mRESIST, for overall response, the ORR was $38 \%$ in the HAICAP group and $30 \%$ in the HAIC group ( $\mathrm{p}=$ $0.227)$, and the DCR was $83 \%$ in the HAICAP group and $66 \%$ in the HAIC group $(\mathrm{p}=0.006)$. For intrahepatic response, the DCR was higher in the HAICAP group $(85 \%)$ than in the PL group $(74 \%)(p=0.045)$.

\section{Safety}

All AEs were evaluated as mild and manageable, and no toxicity-associated deaths occurred in the follow-up. In both groups, the most common AEs were pain, fever and vomiting. Laboratory tests showed that decreased albumin, and increased alanine aminotransferase and aspartate aminotransferase were the most common laboratory-related changes. No significant difference 
Table 2 Summary of Best Response

\begin{tabular}{|c|c|c|c|}
\hline \multirow[t]{2}{*}{ Variable } & HAICAP $(n=8 I)$ & HAIC $(n=\mid 48)$ & \multirow[t]{2}{*}{$P$ value } \\
\hline & No. $(\%)^{c}$ & No. $(\%)^{c}$ & \\
\hline \multicolumn{4}{|l|}{ Overall Response ${ }^{a}$} \\
\hline Complete response & $0(0)$ & $0(0)$ & 1.000 \\
\hline Partial response & $31(38)$ & $45(30)$ & 0.227 \\
\hline Stable response & $36(44)$ & $52(35)$ & 0.166 \\
\hline Progressive response & $12(15)$ & $38(26)$ & 0.057 \\
\hline Not assessable & $2(2)$ & $13(8)$ & 0.092 \\
\hline Overall response rate & $31(38)$ & $45(30)$ & 0.227 \\
\hline Disease control rate & $67(83)$ & $97(66)$ & 0.006 \\
\hline \multicolumn{4}{|c|}{ Intrahepatic Response ${ }^{b}$} \\
\hline Complete response & $0(0)$ & $0(0)$ & 1.000 \\
\hline Partial response & $33(4 I)$ & $53(36)$ & 0.461 \\
\hline Stable response & $36(44)$ & $56(38)$ & 0.576 \\
\hline Progressive response & $10(12)$ & $26(18)$ & 0.299 \\
\hline Not assessable & $2(2)$ & $13(8)$ & 0.092 \\
\hline Overall response rate & $33(4 I)$ & $53(36)$ & $0.46 I$ \\
\hline Disease control rate & $69(85)$ & $109(74)$ & 0.045 \\
\hline
\end{tabular}

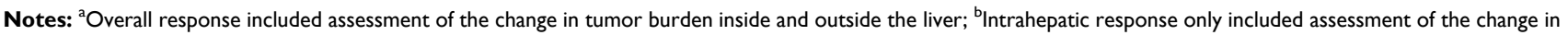
tumor burden inside the liver; 'Treatment response was assessed in evaluable patients.

Abbreviations: HAIC, hepatic infusion chemotherapy; HAIC + AP, hepatic infusion chemotherapy plus anti-programmed cell death protein I.

was found in terms of AEs rate between the two groups. The details of the events were summarized in Supplementary Table S3.

\section{Prognostic Factor Analysis}

The prognostic factors for survival are shown in Table 3. The comparison of HAICAP to HAIC was identified as an independent risk factor for both $\mathrm{OS}(\mathrm{HR}=0.518 ; 95 \% \mathrm{CI}$ $0.314-0.854 ; \mathrm{p}=0.010)$ and PFS $(\mathrm{HR}=0.617 ; 95 \% \mathrm{CI}$ $0.435-0.876 ; \mathrm{p}=0.007)$ in multivariate analysis.

\section{Discussion}

Treatment strategies for advanced HCC have progressed with the emergence of updated technology of intervention chemotherapy and immune-targeted therapy. This retrospective study demonstrated anti-PD-1 immunotherapy combined with HAIC can achieve a better survival benefit and tumor control rate compare to HAIC alone for patients with advanced HCC.

HAIC is frequently adopted as a treatment for locally advanced HCC in Japan and other Asian countries. Its reported median OS is 2.8 to 15.9 months, and a median time to progression of 2.0 to 7.5 months. ${ }^{20}$ The survival outcomes were consistent in our study. Several studies have investigated the efficacy of HAIC-related combination therapies in advanced HCC. In the same center, $\mathrm{He}$ et al reported that HAIC plus sorafenib showed a median OS of 13.37 months and a median PFS of 7.03 months in HCC patients with portal vein tumor thrombus (PVTT). ${ }^{21}$ In addition, a randomized, Phase II trial in Japan revealed sequential HAICsorafenib regimen failed to improve the survival benefit compared with sorafenib alone for advanced HCC. ${ }^{22}$ However, the study was underpowered regarding its primary and secondary endpoints. Another study reported that HAIC plus radiotherapy brought a significantly longer OS and PFS (median, 9.9 vs 5.3, and 3.9 vs 1.9 months) compared to sorafenib alone in HCCs with main PVTT. ${ }^{23}$ In contrast, HAIC plus PD-1 inhibitors in our study brought satisfying survival benefits and had the potential to be an option in the treatment of advanced HCC.

In the subgroup analysis, significant differences were not reached in certain subgroups with small proportional cohorts due to limitations in the number of cases. In general, HAICAP versus HAIC provided a survival advantage in patients with multiple, bi-lobar tumors and tumor diameters greater than $10 \mathrm{~cm}$. It should be noted, however, that HAICAP was less effective in patients with main portal vein tumor thrombus and 


\begin{tabular}{|c|c|c|c|c|c|c|c|c|c|c|c|c|c|c|c|c|c|c|c|c|c|c|}
\hline \multirow{6}{*}{ 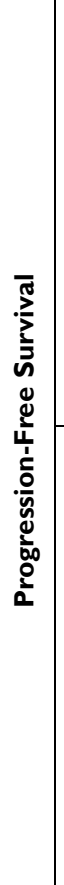 } & \multirow{3}{*}{ 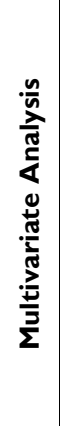 } & $a$ & & & & & & & & & & $\overline{8}$ & & & & & & & & & o̊ & 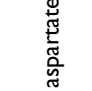 \\
\hline & & $\begin{array}{l}\bar{u} \\
\text { ํํ } \\
\text { ฉू }\end{array}$ & & & & & & & & & & 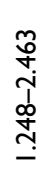 & & & & & & & & & $\begin{array}{c}0 \\
\infty \\
0 \\
0 \\
\omega \\
\tilde{m} \\
0 \\
0\end{array}$ & \\
\hline & & $\stackrel{\propto}{I}$ & & & & & & & & & & $\stackrel{n}{\stackrel{n}{\underline{n}}}$ & & & & & & & & & $\frac{1}{0}$ & \\
\hline & & $a$ & 疋 & $\begin{array}{l}\infty \\
\infty \\
0 \\
0\end{array}$ & 告 & $\frac{\overline{0}}{0}$ & $\frac{5}{0}$ & 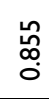 & 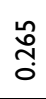 & 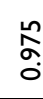 & 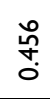 & $\overline{0}$ & ồ. & 岕 & $\frac{\text { I }}{0}$ & $\frac{0}{0}$ & 翣 & 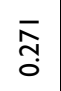 & I $\begin{array}{c}\text { on } \\
0 \\
0\end{array}$ & 官 & $\stackrel{\circ}{\circ}$ & \\
\hline & 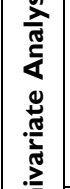 & $\begin{array}{l}\bar{u} \\
\text { ํํ }\end{array}$ & $\begin{array}{l}\frac{0}{0} \\
\vdots \\
m \\
\tilde{m} \\
\stackrel{0}{0}\end{array}$ & 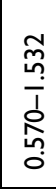 & 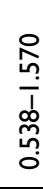 & $\begin{array}{l}\stackrel{\infty}{ઈ} \\
\frac{1}{1} \\
\delta \\
\vdots \\
0\end{array}$ & $\begin{array}{l}0 \\
0 \\
0 \\
1 \\
0 \\
0 \\
0 \\
0 \\
0 \\
0\end{array}$ & \begin{tabular}{l}
\multirow{3}{*}{} \\
$\frac{1}{1}$ \\
$\frac{1}{t}$ \\
\multirow{1}{0}{} \\
0
\end{tabular} & 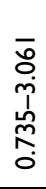 & 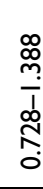 & 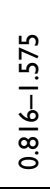 & 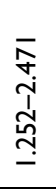 & 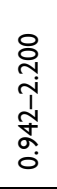 & $\begin{array}{l}0 \\
o \\
i \\
b \\
\frac{1}{0} \\
0\end{array}$ & $\begin{array}{l}\stackrel{n}{0} \\
\infty \\
\vdots \\
\tilde{\alpha} \\
\sigma \\
0\end{array}$ & $\begin{array}{l}\frac{1}{2} \\
\frac{1}{1} \\
0 \\
0\end{array}$ & 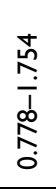 & $\begin{array}{l}0 \\
\stackrel{0}{0} \\
i \\
0 \\
\infty \\
0\end{array}$ & 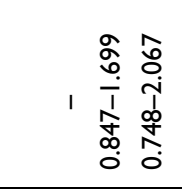 & 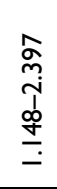 & $\begin{array}{l}\mathbb{N} \\
\infty \\
0 \\
1 \\
m \\
\tilde{m} \\
0\end{array}$ & \\
\hline & & $\stackrel{\mathscr{C}}{\boldsymbol{I}}$ & 尺̊ & $\begin{array}{l}\text { ñ } \\
\text { o. } \\
0\end{array}$ & $\frac{a}{\sigma}$ & $\stackrel{\nwarrow ָ}{\underline{I}}$ & $\begin{array}{l}8 \\
\stackrel{2}{0} \\
0\end{array}$ & 弚 & 总 & 옹 & $\stackrel{m}{\underline{m}}$ & $\stackrel{\stackrel{a}{\stackrel{n}{=}}}{=}$ & 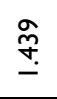 & $\stackrel{\underline{\delta}}{=}$ & $\overline{\bar{m}}$ & 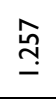 & $\stackrel{\infty}{\underline{0}}$ & 官 & 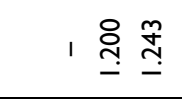 & $\stackrel{\substack{0 \\
0}}{-}$ & $\frac{20}{0}$ & \\
\hline \multirow{6}{*}{ 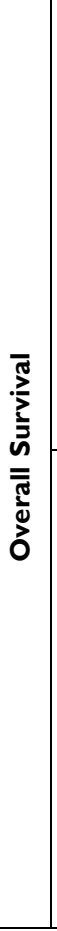 } & \multirow{3}{*}{ 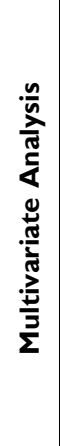 } & $\begin{array}{l}\frac{0}{2} \\
\frac{2}{2} \\
0\end{array}$ & & & & & & & & & రั & $\overline{\bar{o}}$ & & & & & & & $\frac{a}{\bar{a}} \stackrel{0}{0}$ & & 응 & \\
\hline & & $\begin{array}{l}\bar{u} \\
\text { ํํ }\end{array}$ & & & & & & & & & 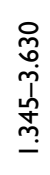 & 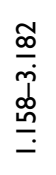 & & & & & & & 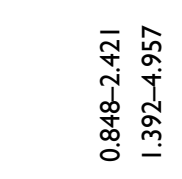 & & $\begin{array}{l}\text { 吕 } \\
0 \\
o \\
j \\
\frac{j}{m} \\
0\end{array}$ & $\tilde{J}$ \\
\hline & & $\stackrel{\mathscr{C}}{\boldsymbol{I}}$ & & & & & & & & & $\frac{0}{i}$ & 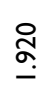 & & & & & & & 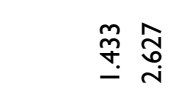 & & $\begin{array}{l}\frac{\infty}{n} \\
0\end{array}$ & \\
\hline & \multirow{3}{*}{ 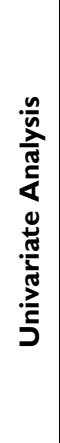 } & $a$ & ્ָ & $\frac{a}{\hat{\alpha}}$ & $\stackrel{\stackrel{m}{m}}{0}$ & $\overline{0}$ & 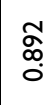 & o̊ & $\underset{\substack{a \\
\infty \\
0 \\
0}}{0}$ & $\stackrel{m}{\tilde{f}}$ & $\overline{8}$ & రి & $\frac{0}{0}$ & $\frac{\circ}{\frac{m}{0}}$ & ח̊̊. & $\frac{1}{0}$ & fó & $\stackrel{a}{0}$ & । & ठั & $\frac{\infty}{0}$ & \\
\hline & & $\begin{array}{l}\bar{U} \\
\text { ํํ }\end{array}$ & $\frac{8}{\frac{8}{1}}$ & $\begin{array}{l}0 \\
0 \\
0 \\
\vdots \\
1 \\
0 \\
0 \\
0 \\
0\end{array}$ & $\begin{array}{l}\frac{0}{2} \\
\text { j. } \\
0 \\
\stackrel{0}{0} \\
0\end{array}$ & 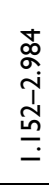 & 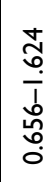 & 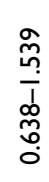 & 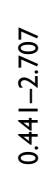 & $\begin{array}{l}o \\
o \\
o \\
o \\
o \\
0 \\
0\end{array}$ & $\begin{array}{l}\frac{m}{R} \\
\dot{p} \\
\dot{p} \\
\dot{o} \\
\dot{q}\end{array}$ & 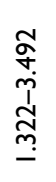 & $\begin{array}{l}\hat{y} \\
\stackrel{y}{+} \\
\underline{0} \\
=\end{array}$ & 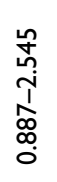 & $\begin{array}{l}\overline{3} \\
\stackrel{0}{0} \\
\tilde{d} \\
\stackrel{m}{-}\end{array}$ & 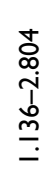 & 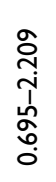 & 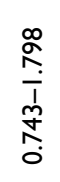 & 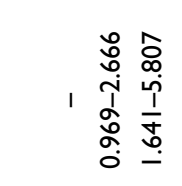 & 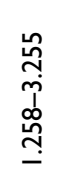 & $\begin{array}{l}\hat{o} \\
0 \\
0 \\
0 \\
\text { s. } \\
m \\
0\end{array}$ & \\
\hline & & $\frac{\mathscr{c}}{I}$ & ô & 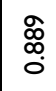 & 节 & 芯 & $\tilde{\tilde{o}}$ & $\begin{array}{l}\bar{\alpha} \\
\text { o. }\end{array}$ & ơ & $\stackrel{2}{=}$ & 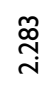 & $\frac{q}{\dot{v}}$ & 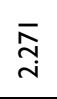 & 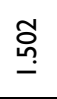 & $\frac{t}{i}$ & $\stackrel{\stackrel{\leftrightarrow}{\infty}}{=}$ & 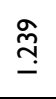 & $\stackrel{\stackrel{\circ}{ }}{=}$ & 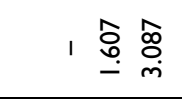 & 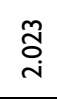 & 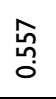 & \\
\hline 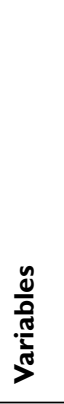 & & & 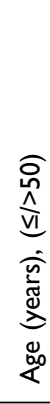 & 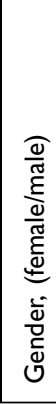 & 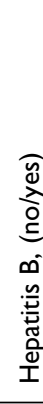 & 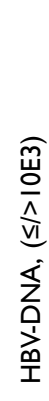 & 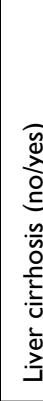 & $\begin{array}{l}\tilde{\hat{n}} \\
\hat{\underline{v}} \\
\tilde{c}\end{array}$ & 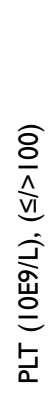 & 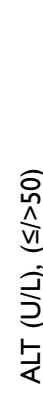 & 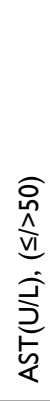 & 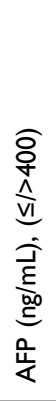 & 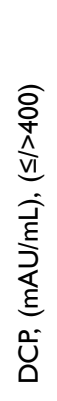 & 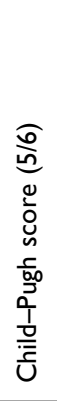 & 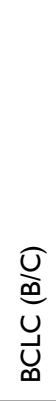 & 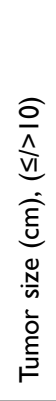 & 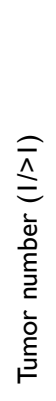 & 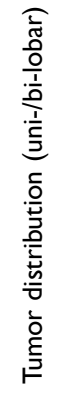 & 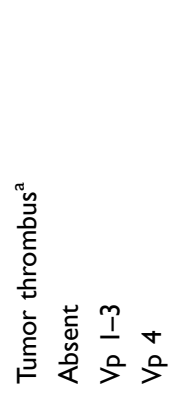 & 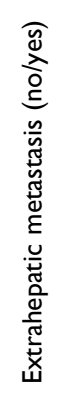 & 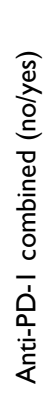 & $\begin{array}{l}\text { s. } \\
\text { r. }\end{array}$ \\
\hline
\end{tabular}


extrahepatic metastases. These findings suggested that as a locoregional approach, HAIC can control intrahepatic lesions. It is, unfortunately, not as effective in managing extrahepatic metastases. Although anti-PD-1 therapy was combined, it might only produce limited benefit for survival in very late-stage $\mathrm{HCC}$ patients. However, the treatment response showed higher DCR in the HAICAP group compared to the HAIC group, especially in overall response analysis (83\% vs $66 \%)$. Given HAIC has limited ability to control the progression of extrahepatic metastases, anti-PD-1 therapy mobilizes systemic immune response, which may compensate for the limitation of HAIC monotherapy.

Of note, all the patients in our study received HAIC of the oxaliplatin-based regime, which is more effective than cisplatin-based regime. ${ }^{21}$ The survival benefit observed in this study may owe to the synergistic antitumor effect of PD-1 inhibitors and Oxaliplatin. 5-Fluorouracil combined with oxaliplatin can induce immunogenic cell death (ICD) in the murine colon cancer model and improve anti-PD-1 efficacy, reversing the resistance of colon cancer to immunotherapy. ${ }^{24}$ Besides, oxaliplatin can be used as an inducer of ICD and as a modulator of the tumor immune microenvironment. Combination of PD-1 inhibitors enhances the suppression of HCC growth and TGF- $\beta$ secretion and augmentation of inflammatory cytokine secretion. $^{25}$

The present study has some limitations. First, the study was a retrospective study in a single center. A majority of included patients were with hepatitisB-related HCC. The results need to be validated in external cohort with different disease backgrounds. Second, the categories of PD-1 inhibitors varied in the HAICAP group, which might influence the uniformity of the treatment procedure. Third, due to the limitations of retrospective study, subsequent therapies after HAIC or HAICAP were uncontrolled. Treatment options have an impact on survival to some extend. Findings from this study should be further expanded to a multicenter study to obtain higher level medical evidence.

\section{Conclusion}

Based on our results, HAICAP is associated with better treatment response and survival benefits compared to HAIC. Thus, HAICAP may be a potential new treatment option for advanced HCC.

\section{Data Sharing Statement}

The data are available from the Sun Yat-sen University Cancer Center Institutional Data Access/Ethics Committee for researchers who meet the criteria for access to the confidential data.

\section{Ethics Approval Statement}

This study was conducted according to the ethical guidelines of the 1975 Declaration of Helsinki. This research was approved by the institutional review board of Sun Yat-sen University Cancer Center (RDDA2020001831). Patient informed consent is exempt because the study used retrospective anonymous clinical data that were obtained after each patient agreed to treatment. Individuals cannot be identified based on the data presented. We declare to ensure the confidentiality of patient data.

\section{Acknowledgments}

The authors thank Malaysian Professor Anna Kan (annakan@sysucc.org.cn) for English language polishing.

\section{Author Contributions}

All authors made a significant contribution to the work reported, whether that is in the conception, study design, execution, acquisition of data, analysis and interpretation, or in all these areas; took part in drafting, revising or critically reviewing the article; gave final approval of the version to be published; have agreed on the journal to which the article has been submitted; and agree to be accountable for all aspects of the work.

\section{Funding}

There is no funding to report.

\section{Disclosure}

The authors have no conflicts of interest to declare.

\section{References}

1. Bray F, Ferlay J, Soerjomataram I, Siegel RL, Torre LA, Jemal A. Global cancer statistics 2018: GLOBOCAN estimates of incidence and mortality worldwide for 36 cancers in 185 countries. CA Cancer J Clin. 2018;68(6):394-424. doi:10.3322/caac.21492

2. Llovet JM, Ricci S, Mazzaferro V, et al. Sorafenib in advanced hepatocellular carcinoma. $N$ Engl J Med. 2008;359(4):378-390. doi:10.1056/NEJMoa0708857

3. Cheng AL, Kang YK, Chen Z, et al. Efficacy and safety of sorafenib in patients in the Asia-Pacific region with advanced hepatocellular carcinoma: a Phase III randomised, double-blind, placebo-controlled trial. Lancet Oncol. 2009;10(1):25-34. doi:10.1016/S1470-2045(08) 70285-7 
4. Lyu N, Lin Y, Kong Y, et al. FOXAI: a phase II trial evaluating the efficacy and safety of hepatic arterial infusion of oxaliplatin plus fluorouracil/leucovorin for advanced hepatocellular carcinoma. Gut. 2018;67(2):395-396. doi:10.1136/gutjnl-2017-314138

5. Obi S, Sato S, Kawai T. Current status of hepatic arterial infusion chemotherapy. Liver Cancer. 2015;4(3):188-199. doi:10.1159/ 000367746

6. Kudo M, Matsui O, Izumi N, et al. JSH consensus-based clinical practice guidelines for the management of hepatocellular carcinoma: 2014 update by the liver cancer study group of Japan. Liver Cancer. 2014;3(3-4):458-468. doi:10.1159/000343875

7. Lyu N, Kong Y, Mu L, et al. Hepatic arterial infusion of oxaliplatin plus fluorouracil/leucovorin vs. sorafenib for advanced hepatocellular carcinoma. J Hepatol. 2018;69(1):60-69. doi:10.1016/j. jhep.2018.02.008

8. Liu M, Shi J, Mou T, et al. Systematic review of hepatic arterial infusion chemotherapy versus sorafenib in patients with hepatocellular carcinoma with portal vein tumor thrombosis. J Gastroenterol Hepatol. 2020;35(8):1277-1287. doi:10.1111/jgh.15010

9. He MK, Le Y, Li QJ, et al. Hepatic artery infusion chemotherapy using mFOLFOX versus transarterial chemoembolization for massive unresectable hepatocellular carcinoma: a prospective non-randomized study. Chin J Cancer. 2017;36(1):83. doi:10.1186/s40880-017-02512

10. Tsai WL, Lai KH, Liang HL, et al. Hepatic arterial infusion chemotherapy for patients with huge unresectable hepatocellular carcinoma. PLoS One. 2014;9(5):e92784. doi:10.1371/journal. pone.0092784

11. Tsai WL, Sun WC, Chen WC, et al. Hepatic arterial infusion chemotherapy vs transcatheter arterial embolization for patients with huge unresectable hepatocellular carcinoma. Medicine. 2020;99(32): e21489. doi:10.1097/MD.0000000000021489

12. Ueshima K, Ogasawara S, Ikeda M, et al. Hepatic arterial infusion chemotherapy versus sorafenib in patients with advanced hepatocellular carcinoma. Liver Cancer. 2020;9(5):583-595. doi:10.1159/ 000508724

13. Yau T, Hsu C, Kim TY, et al. Nivolumab in advanced hepatocellular carcinoma: sorafenib-experienced Asian cohort analysis. J Hepatol. 2019;71(3):543-552. doi:10.1016/j.jhep.2019.05.014

14. Finn RS, Ryoo BY, Merle P, et al. Pembrolizumab as second-line therapy in patients with advanced hepatocellular carcinoma in KEYNOTE-240: a randomized, double-blind, phase III trial. J Clin Oncol. 2020;38(3):193-202. doi:10.1200/JCO.19.01307

15. Chiang CL, Chan ACY, Chiu KWH, Kong FS. Combined stereotactic body radiotherapy and checkpoint inhibition in unresectable hepatocellular carcinoma: a potential synergistic treatment strategy. Front Oncol. 2019;9:1157. doi:10.3389/fonc.2019.01157
16. Xu J, Zhang Y, Jia R, et al. Anti-PD-1 antibody SHR-1210 combined with apatinib for advanced hepatocellular carcinoma, gastric, or esophagogastric junction cancer: an open-label, dose escalation and expansion study. Clin Cancer Res. 2019;25(2):515-523. doi:10.1158/1078-0432.CCR-18-2484

17. Bruix J, Reig M, Sherman M. Evidence-based diagnosis, staging, and treatment of patients with hepatocellular carcinoma. Gastroenterology. 2016;150(4):835-853. doi:10.1053/j. gastro.2015.12.041

18. Li S, Mei J, Wang Q, et al. Postoperative adjuvant transarterial infusion chemotherapy with FOLFOX could improve outcomes of hepatocellular carcinoma patients with microvascular invasion: a preliminary report of a phase III, randomized controlled clinical trial. Ann Surg Oncol. 2020. doi:10.1245/s10434-020-08601-8

19. Llovet JM, Lencioni R. mRECIST for HCC: performance and novel refinements. $J$ Hepatol. 2020;72(2):288-306. doi:10.1016/j. jhep.2019.09.026

20. Ikeda M, Morizane C, Ueno M, Okusaka T, Ishii H, Furuse J. Chemotherapy for hepatocellular carcinoma: current status and future perspectives. Jpn J Clin Oncol. 2018;48(2):103-114. doi:10.1093/ jjco/hyx 180

21. He M, Li Q, Zou R, et al. Sorafenib plus hepatic arterial infusion of oxaliplatin, fluorouracil, and leucovorin vs sorafenib alone for hepatocellular carcinoma with portal vein invasion: a randomized clinical trial. JAMA Oncol. 2019. doi:10.1001/jamaoncol.2019.0250

22. Kondo M, Morimoto M, Kobayashi S, et al. Randomized, phase II trial of sequential hepatic arterial infusion chemotherapy and sorafenib versus sorafenib alone as initial therapy for advanced hepatocellular carcinoma: SCOOP-2 trial. BMC Cancer. 2019;19(1):954. doi:10.1186/s12885-019-6198-8

23. Kodama K, Kawaoka T, Aikata H, et al. Comparison of outcome of hepatic arterial infusion chemotherapy combined with radiotherapy and sorafenib for advanced hepatocellular carcinoma patients with major portal vein tumor thrombosis. Oncology. 2018;94(4):215-222. doi:10.1159/000486483

24. Limagne E, Thibaudin M, Nuttin L, et al. Trifluridine/tipiracil plus oxaliplatin improves PD-1 blockade in colorectal cancer by inducing immunogenic cell death and depleting macrophages. Cancer Immunol Res. 2019;7(12):1958-1969. doi:10.1158/2326-6066.CIR19-0228

25. Zhu H, Shan Y, Ge K, Lu J, Kong W, Jia C. Oxaliplatin induces immunogenic cell death in hepatocellular carcinoma cells and synergizes with immune checkpoint blockade therapy. Cell Oncol (Dordr). 2020;43(6):1203-1214. doi:10.1007/s13402-020-00552-2
Journal of Hepatocellular Carcinoma

\section{Publish your work in this journal}

The Journal of Hepatocellular Carcinoma is an international, peerreviewed, open access journal that offers a platform for the dissemination and study of clinical, translational and basic research findings in this rapidly developing field. Development in areas including, but not limited to, epidemiology, vaccination, hepatitis therapy, pathology and molecular tumor classification and prognostication are all considered for publication. The manuscript management system is completely online and includes a very quick and fair peer-review system, which is all easy to use. Visit http://www.dovepress.com/ testimonials.php to read real quotes from published authors. 